\title{
Moving toward a better understanding of radioiodine action
}

\author{
Alan L. Ho \\ Received: 21 May 2013/ Accepted: 25 July 2013/Published online: 30 August 2013 \\ (C) Springer Science+Business Media New York 2013
}

The selective tropism for thyroid tissue makes radioiodine (RAI or ${ }^{131} \mathrm{I}$ ) a unique targeted therapy for both benign and malignant thyroid disorders. The efficacy of ${ }^{131} \mathrm{I}$ is reliant upon uptake and retention of the isotope in thyrocytes to achieve doses of ionizing radiation sufficient to elicit clinically meaningful cytotoxicity. The processes that govern this can be impaired, eliminating ${ }^{131}$ I effectiveness or requiring the administration of higher activity that can lead to greater toxicity. Gaining insight into biologic mechanisms of ${ }^{131} \mathrm{I}$ action is critical for rationally devising therapeutic strategies to circumvent the limitations to ${ }^{131} \mathrm{I}$ therapy that are commonly encountered clinically.

In this issue of endocrine, Russo et al. [1] evaluate the mechanism of ${ }^{131}$ I-mediated cytotoxicity. The investigators approached this question by developing a method for culturing small thyroid tissues for up to 3 weeks in vitro. The advantage of this approach is the preservation of some of the three-dimensional follicular architecture that better represents the spatial considerations relevant in vivo. Using this model, the investigators observed that ${ }^{131} \mathrm{I}$ induced apoptosis 3 days after isotope addition. Presumably, apoptosis in this scenario is a cellular response to DNA damage incurred by ionizing radiation. Greater insight into how known DNA damage pathways may modulate this response in thyrocytes could potentially generate new hypotheses for how to increase ${ }^{131} \mathrm{I}$ efficacy and/or diminish the activity required to generate therapeutic response.

Understanding how ${ }^{131} \mathrm{I}$ elicits cytotoxicity is also relevant for informing the therapeutic use of ${ }^{131} \mathrm{I}$ for recurrent

\footnotetext{
A. L. Ho $(\bowtie)$

Department of Medicine, Memorial Sloan Kettering Cancer Center and Weill Cornell Medical College, New York, NY, USA

e-mail: hoa@mskcc.org
}

and/or metastatic differentiated thyroid cancers. RAIrefractory (RAIR) thyroid cancer continues to be a challenging clinical problem. Without ${ }^{131} \mathrm{I}$, the continuous administration of palliative chemotherapy remains the only systemic option for these patients, which can be associated with cumulative toxicity and acquired drug resistance. It is conceivable that alterations in the DNA damage response presumably necessary for the ${ }^{131} \mathrm{I}$ induced apoptosis observed by Russo and colleagues could serve as a mechanism of resistance in RAIR disease. Much of the research regarding RAIR thyroid cancer, however, has been focused upon the mechanisms by which thyroid tumors lose the ability to concentrate ${ }^{131} \mathrm{I}$. Indeed, the loss of RAI avidity has been correlated to significantly worse prognosis for patients with thyroid cancer [2], and efforts to address ${ }^{131}$ I resistance have largely focused upon developing strategies to restore RAI incorporation in tumors. The loss of RAI avidity has been associated with diminished expression of a subset of thyroid specific genes, including the sodium iodide symporter (NIS) which mediates iodide uptake into thyroid cells. Strategies to "redifferentiate" tumors and restore expression of these key genes to enhance RAI incorporation have been attempted, but unfortunately yielded at best only modest benefit [3-6].

More recently, significant progress has been made with the recognition from several groups that the aberrant activation of the mitogen-activated protein kinase (MAPK) signaling pathway that occurs in about $70 \%$ of papillary thyroid cancers (PTC) via mutually exclusive genetic alterations (i.e., $R E T, N T R K, R A S$, and $B R A F$ ) leads to the diminished expression of genes that govern RAI incorporation in tumors. Pharmacologic inhibition of the pathway in a murine model of PTC driven by oncogenic BRAF successfully restored NIS expression and RAI avidity [7]. Based on this, a pilot study was performed to test if 
inhibition of the MAPK pathway with the MEK 1/2 inhibitor selumetinib (AstraZeneca) can clinically restore RAI avidity and efficacy for RAIR patients [8]. ${ }^{124}$ I positron emission tomography (PET)/CT scans were utilized to precisely quantify drug-induced changes in iodine incorporation within specific lesions ("lesional dosimetry"). Of 20 RAIR patients, 12 had new or increased ${ }^{124}$ I incorporation after selumetinib. For 8 of those patients, sufficient iodine avidity was achieved to warrant therapy with ${ }^{131} \mathrm{I}$. Interestingly, all 5 patients with NRAS mutant tumors qualified for ${ }^{131} \mathrm{I}$ therapy, whereas only 1 out of $9 B R A F$ mutant patients did. One patient with a tumor harboring a RET rearrangement and another patient with a tumor lacking identifiable pathway alterations also received treatment with ${ }^{131}$ I. Tumor reductions following ${ }^{131} \mathrm{I}$ with decreases in serum thyroglobulin were observed for all patients.

These results provide an important proof of principle that RAI avidity and efficacy can be restored with MAPK pathway inhibition. Still, more definitive clinical trials need to be conducted in order to better understand how this approach may be incorporated into routine clinical care. The heterogeneity of response among patients with distinct tumor genotypes highlights the need to better understand determinants of susceptibility in order to innovate ways to broaden the applicability of this approach to more patients. Development of this treatment strategy would also benefit from greater insights into other critical factors that modify the efficacy of ${ }^{131}$ I therapy beyond those that govern iodine uptake/retention. For example, new approaches to optimize apoptosis induced by ${ }^{131} \mathrm{I}$ and decrease the lesional dose threshold required for cytotoxicity could clinically enhance ${ }^{131}$ I efficacy. Such efforts require the use of disease models from which biologically and clinically sound hypotheses may be developed. This rational, mechanism-based approach will formulate a better understanding of how ${ }^{131} \mathrm{I}$ therapy may be enhanced and more effectively applied.

\section{References}

1. E. Russo, A. Guerra, V. Marotta, A. Faggiano, A. Colao, S. Del Vecchio, M. Tonacchera, M. Vitale, Radioiodide induces apoptosis in human thyroid tissue in culture. Endocrine (2013). doi:10. 1007/s12020-013-9940-z

2. C. Durante, N. Haddy, E. Baudin, S. Leboulleux, D. Hartl, J.P. Travagli, B. Caillou, M. Ricard, J.D. Lumbroso, F. De Vathaire, M. Schlumberger, Long-term outcome of 444 patients with distant metastases from papillary and follicular thyroid carcinoma: benefits and limits of radioiodine therapy. J. Clin. Endocrinol. Metab. 91, 2892-2899 (2006)

3. D. Handkiewicz-Junak, J. Roskosz, K. Hasse-Lazar, S. SzpakUlczok, Z. Puch, A. Kukulska, T. Olczyk, A. Piela, E. PaliczkaCieslik, B. Jarzab, 13-cis-retinoic acid re-differentiation therapy and recombinant human thyrotropin-aided radioiodine treatment of non-Functional metastatic thyroid cancer: a single-center, 53-patient phase 2 study. Thyroid Res. 2, 8 (2009)

4. E.J. Sherman, Y.B. Su, A. Lyall, H. Schoder, M.G. Fury, R.A. Ghossein, S. Haque, D. Lisa, A.R. Shaha, R.M. Tuttle, D.G. Pfister, Evaluation of romidepsin for clinical activity and radioactive iodine reuptake in radioactive iodine-refractory thyroid carcinoma. Thyroid 23, 593-599 (2013)

5. D. Simon, C. Korber, M. Krausch, J. Segering, P. Groth, R. Gorges, F. Grunwald, H.W. Muller-Gartner, C. Schmutzler, J. Kohrle, H.D. Roher, C. Reiners, Clinical impact of retinoids in redifferentiation therapy of advanced thyroid cancer: final results of a pilot study. Eur. J. Nucl. Med. Mol. Imaging 29, 775-782 (2002)

6. J.A. Woyach, R.T. Kloos, M.D. Ringel, D. Arbogast, M. Collamore, J.A. Zwiebel, M. Grever, M. Villalona-Calero, M.H. Shah, Lack of therapeutic effect of the histone deacetylase inhibitor vorinostat in patients with metastatic radioiodine-refractory thyroid carcinoma. J. Clin. Endocrinol. Metab. 94, 164-170 (2009)

7. D. Chakravarty, E. Santos, M. Ryder, J.A. Knauf, X.H. Liao, B.L. West, G. Bollag, R. Kolesnick, T.H. Thin, N. Rosen, P. Zanzonico, S.M. Larson, S. Refetoff, R. Ghossein, J.A. Fagin, Small-molecule MAPK inhibitors restore radioiodine incorporation in mouse thyroid cancers with conditional BRAF activation. J. Clin. Invest. 121, 4700-4711 (2011)

8. A.L. Ho, R.K. Grewal, R. Leboeuf, E.J. Sherman, D.G. Pfister, D. Deandreis, K.S. Pentlow, P.B. Zanzonico, S. Haque, S. Gavane, R.A. Ghossein, J.C. Ricarte-Filho, J.M. Dominguez, R. Shen, R.M. Tuttle, S.M. Larson, J.A. Fagin, Selumetinib-enhanced radioiodine uptake in advanced thyroid cancer. N. Engl. J. Med. 368, 623-632 (2013) 